\title{
On typical parametrizations of finite-dimensional compacta on the Cantor set
}

\author{
by
}

\author{
Paweł Milewski (Warszawa)
}

\begin{abstract}
We prove that if $X$ is a perfect finite-dimensional compactum, then for almost every continuous surjection of the Cantor set onto $X$, the set of points of maximal order is uncountable. Moreover, if $X$ is a perfect compactum of positive finite dimension then for a typical parametrization of $X$ on the Cantor set, the set of points of maximal order is homeomorphic to the product of the rationals and the Cantor set.
\end{abstract}

1. Introduction. All topological spaces considered in this paper are separable metrizable. By a compactum we mean a compact metrizable space. Each compactum $X$ has a parametrization on the Cantor set $2^{\infty}$, i.e., a continuous surjection $f: 2^{\infty} \rightarrow X$. The order of $x \in X$ is the cardinality $\left|f^{-1}(x)\right|$ of the fiber, and the order of $f$ is the supremum of the orders of points in $X$.

The Cantor step function from the ternary Cantor set onto the unit interval $I$ is of order 2 and the points of order 2 form a countable set in $I$. It follows from the results of this paper that, from the point of view of the Baire category in the function space, this is not a typical situation.

This is a particular instance of the following phenomenon. A compactum $X$ is rational (respectively, of rational dimension $\leq n$ ) if there is a countable set $E \subset X$ such that $X \backslash E$ is zero-dimensional (resp. $\operatorname{dim}(X \backslash E) \leq n-1$ ) (cf. $[\mathrm{Ku}, 51, \mathrm{I}],[\mathrm{No}]$ ). The interval $I$ is rational and the $n$-cube $I^{n}$ is of rational dimension $\leq n$. A theorem of Lelek and Mohler $[\mathrm{LM}]$ asserts that in the space of parametrizations of a perfect rational compactum $X$ on $2^{\infty}$, the parametrizations of order 2 with countable set of points having the maximal order form a dense set. Similarly, for any compactum $X$ with rational dimension $\leq n$, the parametrizations of order $\leq n+1$ with at most countably many points of order $n+1$ are dense in the space of parametrizations

2000 Mathematics Subject Classification: 54D30, 54C50.

Key words and phrases: dimension, compact, parametrization, residual. 
of $X$ on $2^{\infty}$ (cf. [PPR, Comment 9.6]). Nevertheless, such parametrizations always form a meager set in the space of all parametrizations.

Before stating our main result, let us recall Kuratowski's theorem $[\mathrm{Ku}, 45, \mathrm{II}]$ that for any $k$-dimensional compactum $X$ without isolated points, almost every parametrization of $X$ on $2^{\infty}$ has order $k+1$. The terminology is explained in the next section.

1.1. TheOREM. Let $X$ be a $k$-dimensional compactum without isolated points, $k \geq 1$. Then for almost every continuous surjection $f: 2^{\infty} \rightarrow X$ the set of points in $X$ of maximal order is uncountable.

This result can be refined to the following effect.

1.2. Corollary. Let $X$ be a $k$-dimensional compactum, $k \geq 1$. Then for a typical parametrization of $X$ on the Cantor set, the set of points of order $k+1$ is homeomorphic to the product of the rationals and the Cantor set.

There is a connection between our results and an interesting problem about the descriptive class of the family of rational compacta in the hyperspace of the Hilbert cube. We comment on this in Section 5.

One should also mention here a very interesting work by Bruckner and Garg [BG] characterizing typical continuous maps from the unit interval to the real line. The structure of such typical maps is quite different from that we encountered in our case.

The main idea of the proof of Theorem 1.1 is related to the reasoning in $[\mathrm{Po}]$, where parametrizations of infinite-dimensional compacta on the Cantor set were considered.

2. Terminology and auxiliary results. We follow $[\mathrm{Ke}]$ and $[\mathrm{Ku}]$. We denote by $2^{n}$ the set of zero-one sequences of length $n$, i.e., $2^{n}=$ $\left\{\left(i_{0}, \ldots, i_{n-1}\right): i_{k} \in\{0,1\}, k=0, \ldots, n-1\right\}$. The set $2^{0}$ reduces to the empty sequence $\emptyset$. The concatenation of $s=\left(i_{0}, \ldots, i_{n-1}\right)$ and $j \in\{0,1\}$ is the sequence $\hat{s} j=\left(i_{0}, \ldots, i_{n-1}, j\right) \in 2^{n+1}$. The Cantor set $2^{\infty}$ is the set of all infinite zero-one sequences equipped with the pointwise convergence topology.

Given families $\mathcal{E}, \mathcal{F}$ of subsets of a space $X$ we say that $\mathcal{F}$ shrinks $\mathcal{E}$ if each element of $\mathcal{E}$ contains exactly one element of $\mathcal{F}$.

A continuous surjection $f: 2^{\infty} \rightarrow X$ is called a parametrization of $X$. The space $\mathcal{S}\left(2^{\infty}, X\right)$ of all parametrizations of $X$ is equipped with the uniform convergence topology, hence $\mathcal{S}\left(2^{\infty}, X\right)$ is completely metrizable and separable. We denote by $B(f, \varepsilon)$ the $\varepsilon$-ball centered at $f \in \mathcal{S}\left(2^{\infty}, X\right)$ in the supremum metric. For every $f \in \mathcal{S}\left(2^{\infty}, X\right)$ we denote by $X(f, i)$ the set of points $y \in X$ with $\left|f^{-1}(y)\right|=i$ and by $D(f, i)$ the inverse image $f^{-1}[X(f, i)]$. 
Given $f \in \mathcal{S}\left(2^{\infty}, X\right)$ the elements of $X(f, i), i \leq \infty$, are called points of order $i$, and the order of $f$ is the supremum $\sup \{i \leq \infty: X(f, i) \neq \emptyset\}$.

Saying that a typical parametrization has a certain property, or that the property holds for almost every parametrization, means that in the space of all parametrizations the set of all maps with the given property is residual.

The Kuratowski theorem $[\mathrm{Ku}, 45, \mathrm{II}]$ asserts that if $X$ is a $k$-dimensional compactum without isolated points, then the set of parametrizations of order $k+1$ is residual in $\mathcal{S}\left(2^{\infty}, X\right)$. In fact, Kuratowski's proof shows a bit more: if $K$ is a $k$-dimensional perfect closed subset of a compactum $X$, then for a typical parametrization $f$ of $X$, we have $K \subset \bigcup_{i=1}^{k+1} X(f, i)$.

We shall need the following theorem of Alexandroff and Urysohn [AU] (cf. $[\mathrm{vE}])$.

2.1. Theorem (Alexandroff-Urysohn). Every zero-dimensional $\sigma$-compact nowhere compact and nowhere countable space is homeomorphic to the product of the rationals and the Cantor set.

We shall also use a topological characterization of the product of the rationals and the irrationals given by van Mill [vM].

2.2. Theorem (van Mill). The product of the rationals and the irrationals is the unique, up to homeomorphism, zero-dimensional nowhere completely metrizable and nowhere $\sigma$-compact space which is the countable union of completely metrizable closed subsets.

An important role in the proof of Theorem 1.1 is played by the following fact (cf. [En, Theorem 1.6.9]).

2.3. Lemma (Hemmingsen). For every compact subset $S$ of a space $X$, with $\operatorname{dim} S \geq k$, there are open sets $G_{1}, \ldots, G_{k+1}$ in $X$ such that $S \subset$ $G_{1} \cup \ldots \cup G_{k+1}$ and $S \cap L_{1} \cap \ldots \cap L_{k+1} \neq \emptyset$ whenever $L_{1}, \ldots, L_{k+1}$ are closed, $L_{i} \subset G_{i}$ and $S \subset L_{1} \cup \ldots \cup L_{k+1}$.

A subset $A$ of a space $X$ has the Baire property if there is a set $M \subset X$ of first category such that $A=(U \backslash M) \cup(M \backslash U)$ for some open $U \subset X$. A subset $A$ of a completely metrizable space $X$ is analytic if it is a continuous image of the irrationals; the complements of analytic sets are coanalytic. Every analytic set has the Baire property (cf. [Ke, Theorem 21.6]). Thus in order to prove that an analytic set is residual one has to show that it intersects each nonmeager $G_{\delta}$ set.

3. Proof of Theorem 1.1. Let $X$ be as in Theorem 1.1. The assertion follows instantly from the following

3.1. Lemma. Let $H \subset X$ be an open $k$-dimensional set, $k \geq 1$. Then the set 


$$
A(H)=\left\{f \in \mathcal{S}\left(2^{\infty}, X\right): H \cap X(f, k+1) \text { is uncountable }\right\}
$$

is residual in $\mathcal{S}\left(2^{\infty}, X\right)$.

The proof of Lemma 3.1 will be divided into two steps.

SteP I. Let $K$ be the set of $y \in X$ without any neighborhood of dimension less than $k$. Let $f: 2^{\infty} \rightarrow X$ be a parametrization and fix $\varepsilon>0$.

Assume that we are given $(k+1)$-element families $\mathcal{E}_{s}, s \in 2^{n}$, such that $\bigcup\left\{\mathcal{E}_{s}: s \in 2^{n}\right\}$ is a collection of pairwise disjoint clopen subsets of $2^{\infty}$, and there are also points $y_{s}$ with

$$
y_{s} \in K \cap H \cap \bigcap\left\{f[E]: E \in \mathcal{E}_{s}\right\} \quad \text { and } \quad f^{-1}\left(y_{s}\right) \subset \bigcup \mathcal{E}_{s}, \quad s \in 2^{n} .
$$

We shall construct $(k+1)$-element families $\mathcal{E}_{t}, t \in 2^{n+1}$, and a nonempty open set $\mathcal{U} \subset B(f, \varepsilon)$ such that:

(A) $\mathcal{E}_{s^{\hat{j}}}$ shrinks $\mathcal{E}_{s}$ for $s \in 2^{n}, j=0,1$,

(B) if $u \in \mathcal{U}$ then $u\left[2^{\infty} \backslash \bigcup \mathcal{E}_{s}\right] \cap u\left[\bigcup \mathcal{E}_{s^{\wedge} j}\right]=\emptyset$ and $\operatorname{diam}\left(u\left[\mathcal{E}_{s^{\wedge} j}\right]\right)<\varepsilon$ for $s \in 2^{n}, j=0,1$,

(C) for every $u \in \mathcal{U}$ and $t \in 2^{n+1}$ there is $z_{t} \in K \cap H \cap \bigcap\left\{u[F]: F \in \mathcal{E}_{t}\right\}$ with $u^{-1}\left(z_{t}\right) \subset \bigcup \mathcal{E}_{t}$,

(D) $\bigcup\left\{\mathcal{E}_{t}: t \in 2^{n+1}\right\}$ is a family of pairwise disjoint clopen subsets of $2^{\infty}$ with diameter less than $\varepsilon$.

To carry out the construction, we enumerate the elements of $\mathcal{E}_{s}$ as $\left\{C_{s}^{1}, \ldots, C_{s}^{k+1}\right\}, s \in 2^{n}$. For each $C_{s}^{i}$ choose a clopen set $A_{s}^{i}$ such that $f^{-1}\left(y_{s}\right) \cap C_{s}^{i} \subset A_{s}^{i} \subset C_{s}^{i}, \operatorname{diam}\left(f\left[A_{s}^{i}\right]\right)<\varepsilon / 2$ and $f\left[A_{s}^{i}\right] \cap f\left[2^{\infty} \backslash \bigcup \mathcal{E}_{s}\right]=\emptyset$, and consider $\mathcal{A}_{s}=\left\{A_{s}^{1}, \ldots, A_{s}^{k+1}\right\}$. Then

$$
f\left[2^{\infty} \backslash \bigcup \mathcal{E}_{s}\right] \cap f\left[\bigcup \mathcal{A}_{s}\right]=\emptyset, \quad \operatorname{diam}\left(f\left[\bigcup \mathcal{A}_{s}\right]\right)<\varepsilon .
$$

Since $X \backslash f\left[2^{\infty} \backslash \cup \mathcal{A}_{s}\right]$ is a neighborhood of $y_{s} \in K$, and by definition of $K$, there are pairwise disjoint $k$-dimensional open sets $W_{t} \subset H, t \in 2^{n+1}$, such that

$$
f\left[2^{\infty} \backslash \cup \mathcal{A}_{s}\right] \cap\left(W_{s^{\wedge} 0} \cup W_{s^{1} 1}\right)=\emptyset .
$$

Shrinking, if necessary, the sets $W_{s^{\wedge} 0}, W_{s^{r} 1}$ we can also demand that

$$
f\left[\cup \mathcal{A}_{s}\right] \backslash\left(W_{s^{*} 0} \cup W_{s^{\prime} 1}\right) \neq \emptyset .
$$

Divide every $A_{s}^{i}$ into clopen pairwise disjoint nonempty sets $C_{s^{\wedge} 0}^{i}, C_{s^{\prime} 1}^{i}, D_{s}^{i}$ with $\operatorname{diam}\left(C_{s^{\prime} j}^{i}\right) \leq \varepsilon, j=0,1$, and let

$$
\mathcal{E}_{t}=\left\{C_{t}^{1}, \ldots, C_{t}^{k+1}\right\}, t \in 2^{n+1}, \quad \mathcal{D}_{s}=\left\{D_{s}^{1}, \ldots, D_{s}^{k+1}\right\}, s \in 2^{n} .
$$

For each $t \in 2^{n+1}$, fix a compactum $S_{t} \subset W_{t}$ of dimension $k$ and let $G_{t}^{1}, \ldots, G_{t}^{k+1} \subset W_{t}$ be open sets described for $S_{t}$ in Hemmingsen's Lemma 2.3. Let $K_{t}^{i} \subset G_{t}^{i}$ be nonempty compacta such that, for some open sets $V_{t} \subset X$, we have 


$$
S_{t} \subset V_{t} \subset K_{t}^{1} \cup \ldots \cup K_{t}^{k+1} \subset W_{t} .
$$

Note that, by (1), $f\left[\bigcup \mathcal{A}_{s}\right] \backslash\left(V_{s^{\wedge} 0} \cup V_{s^{1} 1}\right) \neq \emptyset$ for $s \in 2^{n}$. Finally, consider a parametrization $g: 2^{\infty} \rightarrow X$ which agrees with $f$ on $2^{\infty} \backslash \bigcup\left\{\bigcup \mathcal{A}_{s}: s \in 2^{n}\right\}$, and satisfies $g\left[\cup \mathcal{D}_{s}\right]=f\left[\cup \mathcal{A}_{s}\right] \backslash\left(V_{s^{\wedge} 0} \cup V_{s^{\prime 1}}\right), g\left[C_{t}^{i}\right]=K_{t}^{i}$ for $t \in 2^{n+1}$, $s \in 2^{n}$.

Then $g$ belongs to the open $\varepsilon$-ball centered at $f$. Since $f$ and $g$ coincide on $2^{\infty} \backslash \bigcup\left\{\bigcup \mathcal{A}_{s}: s \in 2^{n}\right\}$ and $f\left[\bigcup \mathcal{A}_{s}\right]=g\left[\bigcup \mathcal{A}_{s}\right]$ for all $s \in 2^{n}$, we obtain, for $s \in 2^{n}, t \in 2^{n+1}$,

$$
\begin{gathered}
g\left[2^{\infty} \backslash \bigcup \mathcal{E}_{s}\right] \cap g\left[\bigcup \mathcal{E}_{s^{\wedge} j}\right]=\emptyset, \quad j=0,1, \\
g\left[2^{\infty} \backslash \bigcup \mathcal{E}_{t}\right] \cap S_{t}=\emptyset, \\
g\left[C_{t}^{i}\right] \subset G_{t}^{i}, \quad i=1, \ldots, k+1, \\
\operatorname{diam}\left(g\left[\bigcup \mathcal{E}_{t}\right]\right)<\varepsilon .
\end{gathered}
$$

Let $\mathcal{U}$ be a neighborhood of $g$ contained in $B(f, \varepsilon)$, and small enough to ensure that conditions (2)-(5) are satisfied for all $u \in \mathcal{U}$. One readily checks that (A), (B) and (D) are then fulfilled. Let us verify (C). Setting $L_{t}^{i}=u\left[C_{t}^{i}\right]$, we have $L_{t}^{i} \subset G_{t}^{i}$ and since $u\left[2^{\infty}\right]=X$ and $u\left[2^{\infty} \backslash \bigcup \mathcal{E}_{t}\right] \cap S_{t}=\emptyset$, we conclude that $S_{t} \subset L_{t}^{1} \cup \ldots \cup L_{t}^{k+1}$. Therefore for every $t \in 2^{n+1}$ there is $z_{t} \in S_{t} \cap L_{t}^{1} \cap \ldots \cap L_{t}^{k+1} \subset K \cap H \cap \bigcap\left\{u[F]: F \in \mathcal{E}_{t}\right\}$, and (3) yields $u^{-1}\left(z_{t}\right) \subset \bigcup \mathcal{E}_{t}$. This completes the construction in Step I.

STEP II. The set

$$
A(H)=\left\{f \in \mathcal{S}\left(2^{\infty}, X\right): \text { for uncountably many } y \in H,\left|f^{-1}(y)\right|=k+1\right\}
$$

is analytic. Thus, as was explained in Section 2, to prove that it is residual it is sufficient to show that $A(H)$ intersects every $G_{\delta}$ set dense in some open ball in $\mathcal{S}\left(2^{\infty}, X\right)$. Let $G=\bigcap_{n=0}^{\infty} G_{n}$ be a $G_{\delta}$ set dense in $B\left(g_{0}, \varepsilon_{0}\right) \subset$ $\mathcal{S}\left(2^{\infty}, X\right)$, where $G_{0} \supset G_{1} \supset \ldots$ are open. The restriction $g_{0} \mid g_{0}^{-1}[K \cap H]$ is closed and $\operatorname{dim}(K \cap H)=k$, hence there is a point $y_{\emptyset} \in K \cap H$ of order at least $k+1$ (cf. [En, Theorem 1.12.2]). Therefore we can choose a family $\mathcal{E}_{\emptyset}=\left\{C_{\emptyset}^{1}, \ldots, C_{\emptyset}^{k+1}\right\}$ of pairwise disjoint clopen subsets of $2^{\infty}$ such that $\bigcup \mathcal{E}_{\emptyset}=2^{\infty}$ and $y_{\emptyset} \in g_{0}\left[C_{\emptyset}^{1}\right] \cap \ldots \cap g_{0}\left[C_{\emptyset}^{k+1}\right]$. Starting from $\left\langle g_{0}, \mathcal{E}_{\emptyset}, y_{\emptyset}, \varepsilon_{0}\right\rangle$ and applying inductively the result of Step I with $\varepsilon_{n+1}$ decreasing to 0 and smaller than $\operatorname{dist}\left(g_{n+1}\left[2^{\infty} \backslash \bigcup\left\{\bigcup \mathcal{E}_{s}: s \in 2^{n}\right\}\right], g_{n+1}\left[\bigcup\left\{\bigcup \mathcal{E}_{t}: t \in 2^{n+1}\right\}\right]\right)$, we eventually obtain parametrizations $g_{1}, g_{2}, \ldots$ and collections $\mathcal{E}_{s}=\left\{C_{s}^{i}\right.$ : $i=1, \ldots, k+1\}$ of clopen subsets of $2^{\infty}$.

Set $g=\lim _{n} g_{n}$ and consider the $k+1$ pairwise disjoint Cantor sets $C^{i}=\bigcap_{n=0}^{\infty} \bigcup_{s \in 2^{n}} C_{s}^{i}, i=1, \ldots, k+1$. One readily checks that $g\left[C^{1}\right]=$ $\ldots=g\left[C^{k+1}\right] \subset H, g^{-1}\left[g\left[C^{1}\right]\right]=C^{1} \cup \ldots \cup C^{k+1}$ and $g \mid C^{i}$ is an injection for $i=1, \ldots, k+1$. This completes the proof of Lemma 3.1, and hence the proof of Theorem 1.1. 


\section{Typical parametrizations of finite-dimensional compacta. In} this section we give a more exact description of the set of points of order $k+1$ of a typical parametrization of a $k$-dimensional compactum $X$. Assuming additionally that every nonempty open set in $X$ is $k$-dimensional, we shall investigate also the sets of points with fixed order strictly between 1 and $k+1$.

The Kuratowski theorem implies easily that for a typical parametrization of any compactum without isolated points on $2^{\infty}$, the preimage of the set of points of order 1 is residual in $2^{\infty}$. We shall show that an analogous fact is also true if "residual" is replaced by "of full measure" with respect to any fixed Borel measure on $2^{\infty}$. We begin with the refinement of Theorem 1.1.

Proof of Corollary 1.2. Let $K$ be the set described at the beginning of Step I, and let $P$ be the perfect kernel of $X$, i.e., the set of $y \in X$ without any countable neighborhood. Take open subsets $H_{1}, H_{2}, \ldots$ of $X$ such that $K \cap H_{1}, K \cap H_{2}, \ldots$ form a basis of $K$. Let $G$ be the set of parametrizations $f \in \mathcal{S}\left(2^{\infty}, X\right)$ satisfying $X(f, k+1) \cap P \subset K, P \subset X(f, 1) \cup \ldots \cup X(f, k+1)$, and $\left|f^{-1}(y)\right|=1$ for all $y \in X \backslash P$ not isolated in $X$. Using Kuratowski's method $[\mathrm{Ku}, 45, \mathrm{II}]$ one can show that $G$ is residual. Notice that for every $f \in \mathcal{S}\left(2^{\infty}, X\right)$ and any isolated point $y \in X$ we have $\left|f^{-1}(y)\right|=2^{\aleph_{0}}$, so that $X(f, k+1) \subset K$ for all $f \in G$.

By Lemma 3.1, the set $G \cap \bigcap_{i=1}^{\infty} A\left(H_{i}\right)$ is residual. Let $f \in G \cap \bigcap_{i=1}^{\infty} A\left(H_{i}\right)$. Since $f \in G$, the set $X(f, k+1)=P \cap\left\{y \in X:\left|f^{-1}(y)\right| \geq k+1\right\}$ is $\sigma$ compact. ( $P$ is closed and we have $\left|f^{-1}(y)\right| \geq k+1$ iff there are pairwise disjoint $V_{n_{1}}, \ldots, V_{n_{k+1}}$ with $y \in f\left[V_{n_{1}}\right] \cap \ldots \cap f\left[V_{n_{k+1}}\right]$, where $V_{1}, V_{2}, \ldots$ is a basis of $2^{\infty}$.) Since the restriction $f \mid D(f, k+1): D(f, k+1) \rightarrow X(f, k+1)$ is closed and all points of $X(f, k+1)$ are of the same finite order with respect to $f \mid D(f, k+1)$, Nagami's theorem [Na, Theorem 1] ensures that $X(f, k+1)$ is zero-dimensional (cf. also $[\mathrm{Hu}]$ and for more general case [Su]). Therefore, for $f \in G$ the set $X(f, k+1)$ is nowhere compact because it is included and dense in $K$. Finally, since $f \in \bigcap_{i=1}^{\infty} A\left(H_{i}\right)$, the set $X(f, k+1)$ is nowhere countable. Thus, by the Alexandroff-Urysohn theorem, it is homeomorphic to $\mathbb{Q} \times 2^{\infty}$.

4.1. Proposition. If all nonempty open subsets of a compactum $X$ are $k$-dimensional, $k \geq 1$, then for almost every parametrization $f \in \mathcal{S}\left(2^{\infty}, X\right)$ the sets $X(f, 2), \ldots, X(f, k)$ are homeomorphic to $\mathbb{Q} \times \mathcal{N}$ and dense in $X$.

Proof. As was already noticed in the proof of Corollary 1.2, each of the sets $X(f, 2), \ldots, X(f, k)$ is the intersection of a $G_{\delta}$ and an $F_{\sigma}$ set, hence it is the countable union of completely metrizable spaces. By Nagami's theorem [Na, Theorem 1], the sets are zero-dimensional. Let $G \subset \mathcal{S}\left(2^{\infty}, X\right)$ be the set of parametrizations of order $k+1$ and let $f \in G$. The set $X(f, 1)$ is a dense $G_{\delta}$ in $X$, hence each $X(f, i), 1<i \leq k$, is nowhere completely metrizable. 
Since $X$ is the union of zero-dimensional sets $X(f, 1), \ldots, X(f, k+1)$, and, by assumption, every nonempty open subset of $X$ is $k$-dimensional, all the sets $X(f, i), 1 \leq i \leq k$, are nowhere $\sigma$-compact (since $X(f, k+1)$ is $\sigma$-compact). By van Mill's Theorem 2.2 we get the desired conclusion.

As was recalled at the beginning of this section, for a typical parametrization $f$ of a compactum $X$ without isolated points, the sets $D(f, 1)$ and $X(f, 1)$ are dense $G_{\delta}$ in $2^{\infty}$ and $X$, respectively. For measure instead of Baire category, the situation is similar.

4.2. Proposition. Let $X$ be a compactum without isolated points and let $\mu, \nu$ be probability Borel measures on $2^{\infty}$ and $X$, respectively. Then for a typical parametrization $f \in \mathcal{S}\left(2^{\infty}, X\right)$,

(i) $\mu(D(f, 1))=1$,

(ii) $\nu(X(f, 1))=1$.

Proof. First, we prove that

(*) if $T \subset 2^{\infty}$ is nowhere dense then $T \subset D(f, 1)$ for a typical $f \in$ $\mathcal{S}\left(2^{\infty}, X\right)$.

We can assume that $T$ is closed. Then the sets $U_{n}=\left\{f \in \mathcal{S}\left(2^{\infty}, X\right)\right.$ : $\left.\forall x \in T, \operatorname{diam}\left(f^{-1}[f(x)]\right)<1 / n\right\}$ are open for $n \geq 1$.

These sets are also dense in the function space. Indeed, fix $\varepsilon>0, n \geq 1$ and let $f \in \mathcal{S}\left(2^{\infty}, X\right)$. We have to find $g \in B(f, \varepsilon) \cap U_{n}$. To this end take a partition of $2^{\infty}$ into clopen sets $C_{1}, \ldots, C_{m}$ with diameter less than $1 / n$ and such that $\operatorname{diam}\left(f\left[C_{i}\right]\right)<\varepsilon / 3, i=1, \ldots, m$. Divide each $C_{i}$ into nonempty clopen sets $D_{i}, E_{i}$ in such a way that $T \subset D_{1} \cup \ldots \cup D_{m}$. Consider nonempty pairwise disjoint open sets $W_{1}, \ldots, W_{m}$ such that each $W_{i}$ is contained in the $\varepsilon / 3$-neighborhood of $f\left[C_{i}\right]$. It is sufficient to define $g$ by letting $g\left[D_{i}\right]$ $=\left\{d_{i}\right\}$, where $d_{i} \in W_{i}$ are arbitrary, and $g\left[E_{i}\right]=\left(f\left[C_{i}\right] \cup \bar{W}_{i}\right) \backslash \bigcup_{j=1}^{i-1} W_{i}$. Finally, $A=\left\{f \in \mathcal{S}\left(2^{\infty}, X\right): T \subset D(f, 1)\right\}=\bigcap_{n=1}^{\infty} U_{n}$ is a dense $G_{\delta}$ set in $\mathcal{S}\left(2^{\infty}, X\right)$.

We are ready to prove (i). Suppose that there is $\delta>0$ such that the set $B=\left\{f \in \mathcal{S}\left(2^{\infty}, X\right): \mu(D(f, 1)) \leq 1-\delta\right\}$ is not of first category. Take a nowhere dense $T \subset 2^{\infty}$ with $\mu(T)>1-\delta$. By $(\star)$, the set $A$ is residual, hence there exists $f \in A \cap B$, which is, however, impossible.

To show (ii), it is sufficient to apply the Kuratowski theorem and the fact that for every $\varepsilon>0$ there exists a Cantor set $C \subset X$ with $\nu(C)>1-\varepsilon$.

5. Comments. It is an open question whether the family $\mathbf{R}$ of rational compacta in the Hilbert cube $I^{\infty}$ is coanalytic. Let us show a link between this problem and the subject of our paper.

As was already mentioned in Section 1, the set of perfect rational compacta consists exactly of those compacta that admit a parametrization of 
order $\leq 2$ with countably many points of maximal order. Let $x_{0} \in 2^{\infty}$ be an arbitrary point. Using the method of Lelek and Mohler one can show that a compactum $X$ is rational iff there is a nonempty (equivalently, dense) set of parametrizations $f$ of $X \times 2^{\infty}$ such that $\left|f^{-1}(z)\right| \leq 2$ for all $z \in X \times\left\{x_{0}\right\}$ and $\left|f^{-1}(z)\right|=2$ for only countably many $z \in X \times\left\{x_{0}\right\}$. Suppose that there exists a completely metrizable and separable topology on the set of all continuous maps from the Cantor set into the product of the Hilbert cube and the Cantor set, $C\left(2^{\infty}, I^{\infty} \times 2^{\infty}\right)$, such that we can replace "nonempty" by "residual" in the previous statement, and, moreover, suppose that, with respect to this topology, the mapping $\phi: C\left(2^{\infty}, I^{\infty} \times 2^{\infty}\right) \rightarrow \mathcal{K}\left(I^{\infty} \times 2^{\infty}\right)$, given by $\phi(f)=f\left[2^{\infty}\right]$, is open.

Then we could prove the coanalyticity of $\mathbf{R}$ in the following way. Let $\mathcal{R}$ be the set of maps $f \in C\left(2^{\infty}, I^{\infty} \times 2^{\infty}\right)$ such that $f\left[2^{\infty}\right] \cap\left(I^{\infty} \times\left\{x_{0}\right\}\right) \neq \emptyset$, $\left|f^{-1}(z)\right| \leq 2$ for every $z \in I^{\infty} \times\left\{x_{0}\right\}$ and $\left|f^{-1}(z)\right|=1$ for all but countably many $z \in I^{\infty} \times\left\{x_{0}\right\}$. Checking the coanalyticity of $\mathcal{R}$ is standard. By a corollary to the theorem of Lelek and Mohler, $\mathbf{R}=\left\{K \in \mathcal{K}\left(I^{\infty}\right): K \times 2^{\infty}\right.$ $\in \phi[\mathcal{R}]\}$. Applying a theorem of Cenzer and Mauldin $[\mathrm{CM}]$ we infer that $\phi[\mathcal{R}]$ is coanalytic and hence so is $\mathbf{R}$ (as a continuous preimage of a coanalytic set).

Acknowledgements. The author would like to express his thanks to Professor Roman Pol for many valuable conversations on the subject of this paper. The author is also grateful to the referee for remarks which improved the exposition.

\section{References}

[AU] P. Alexandroff und P. Urysohn, Über nulldimensionale Punktmengen, Math. Ann. 98 (1928) 89-106.

[BG] A. M. Bruckner and K. Garg, The level set structure of a residual set of continuous functions, Trans. Amer. Math. Soc. 232 (1977), 307-321.

[CM] D. Cenzer and R. D. Mauldin, Inductive definability: measure and category, Adv. Math. 38 (1980), 55-90.

[vE] A. J. M. van Engelen, Homogeneous Zero-Dimensional Absolute Borel Sets, CWI Tract 27, Stichting Mathematisch Centrum, Centrum voor Wiskunde en Informatica, Amsterdam, 1986.

[En] R. Engelking, Theory of Dimensions, Finite and Infinite, Heldermann, Lemgo, 1995.

[Hu] W. Hurewicz, Über dimensionserhöhende stetige Abbildungen, J. Reine Angew. Math. 169 (1933), 71-78.

[Ke] A. S. Kechris, Classical Descriptive Set Theory, Springer, New York, 1994.

$[\mathrm{Ku}] \quad$ K. Kuratowski, Topology, Vol. II, Academic Press, New York, and PWN, Warszawa, 1968.

[LM] A. Lelek and L. Mohler, On the topology of curves III, Fund. Math. 71 (1971), $147-160$. 
[vM] J. van Mill, Characterization of some zero-dimensional separable metric spaces, Trans. Amer. Math. Soc. 264 (1981), 205-215.

[Na] K. Nagami, Finite-to-one closed mappings and dimension I, Proc. Japan Acad. 34 (1958), 503-506.

[Nö] G. Nöbeling, Über die rationale Dimension, Math. Ann. 109 (1934), 353-375.

[Po] R. Pol, A converse to a theorem of K. Kuratowski on parametrizations of compacta on the Cantor set, Fund. Math. 139 (1991), 37-47.

[PPR] E. Pol, R. Pol and M. Reńska, On countable-dimensional spaces with the Menger property, rational dimension and a question of S. D. Iliadis, Monatsh. Math. 128 (1999), 331-348.

[Su] J. Suzuki, Note on a theorem for dimension, Proc. Japan Acad. 35 (1959), 201-202.

Institute of Mathematics

Warsaw University

Banacha 2

02-097 Warszawa, Poland

E-mail: pamil@mimuw.edu.pl

Received 11 October 2001;

in revised form 7 March 2002 